\title{
THE ALGEBRA GENERATED BY SIMPLE ELEMENTS OF A MATRIX CENTRALIZER
}

\author{
RALPH JOHN DE LA CRUZ
}

Abstract. Let $\mathscr{C}(S)$ denote the centralizer of an arbitrary square matrix $S$. An element $A \in \mathscr{C}(S)$ is simple if $A-I$ is of rank 1 . Let $\mathscr{A}_{S}$ denote the subalgebra generated by the simple elements of $\mathscr{C}(S)$. We use the Weyr canonical form to describe the subalgebra $\mathscr{A}_{S}$, and we show that if $\lambda_{1}, \ldots, \lambda_{k}$ are the distinct eigenvalues of $S$, and $l$ is the number of defective eigenvalues of $S$, then $\mathscr{A}_{S}$ is of dimension $l+\sum_{i=1}^{k} \operatorname{nullity}\left(S-\lambda_{i} I\right)^{2}$.

Mathematics subject classification (2020): 15A03, $15 \mathrm{~A} 27$.

Keywords and phrases: Simple, Weyr structure, Weyr canonical form, centralizer, defective.

\section{REFERENCES}

[1] T. Agapito, A. T. PARAS, $\psi_{S}$-orthogonal matrices and $\psi_{S}$-symmetries, Linear Algebra Appl. 584 (2020) 185-196.

[2] R. J. De la Cruz, K. Dela Rosa, D. I. Merino, A. T. Paras, The Cartan-Diedonne-Scherk Theorems for complex S-orthogonal matrices, Linear Algebra Appl. 458 (2014) 251-260.

[3] M. Catral, L. Lebtahi, J. Stuart, N. Thome, On a matrix group constructed from an $\{R, s+$ $1, k\}$-potent matrix, Linear Algebra Appl. 461 (2014) 200-210.

[4] R. J. DE LA CRUZ, D. I. MERINo, A. T. PARAS, S orthogonal matrices and S symmetries, Linear Algebra Appl. 474 (2015) 213-229.

[5] J. Dieudonne, Sur les generateurs des groupes classiques, Summa Bras. Math. 3 (1955) 149-178.

[6] R. A. Horn And C. R. Johnson, Topics in Matrix Analysis, Cambridge Univesity Press, New York, 1991.

[7] G. HUANG, F. YIN, Constrained inverse eigenproblem and associated approximation problem for anti-Hermitian R-symmetric matrices, Applied Mathematics and Computation 186 (2007) 426-434.

[8] G. HUANG, F. YIN, Matrix inverse problem and its optimal approximation problem for R-symmetric matrices, Applied Mathematics and Computation 189 (2007) 482-489.

[9] K. O’Meara, J. Clark, C. Vinsonhaler, Advanced topics in linear algebra: weaving matrix problems through the Weyr form, Oxford University Press, Oxford 2011.

[10] H. Radjavi, Decomposition of matrices into simple involutions, Linear Algebra Appl., 12 (1975) 247-255.

[11] P. SCHERK, On the decomposition of orthogonalities into symmetries, Proc. Amer. Math. Soc. 1 (1950) 481-491.

[12] W. TRENCH, Characterization and properties of matrices with generalized symmetry or skew symmetry, Linear Algebra Appl. 377 (2004) 207-218.

[13] W. TREnCH, Minimization problems for $(R, S)$-symmetric and $(R, S)$-skew symmetric matrices, Linear Algebra Appl. 389 (2004) 23-31.

[14] F. UHLIG, Constructive ways for generating (generalized) real orthogonal matrices as products of (generalized) symmetries, Linear Algebra Appl. 332-334 (2001) 459-467. 